\title{
Interleaving lattice for the Argonne Advanced Photon Source linac
}

\author{
S. Shin, ${ }^{1, *}$ Y. Sun, ${ }^{2}$ J. Dooling, ${ }^{2}$ M. Borland, ${ }^{2}$ and A. Zholents ${ }^{2}$ \\ ${ }^{1}$ Pohang Accelerator Laboratory, POSTECH, Pohang, Gyungbuk 37673, Korea \\ ${ }^{2}$ Argonne National Laboratory, Argonne, Illinois 60439, USA
}

(Received 4 September 2017; published 12 June 2018)

\begin{abstract}
To realize and test advanced accelerator concepts and hardware, a beam line is being reconfigured in the linac extension area (LEA) of the Argonne Advanced Photon Source (APS) linac. A photocathode rf gun installed at the beginning of the APS linac will provide a low emittance electron beam into the LEA beam line. The thermionic rf gun beam for the APS storage ring and the photocathode rf gun beam for the LEA beam line will be accelerated through the linac in an interleaved fashion. In this paper, the design studies for interleaving lattice realization in the APS linac is described with the initial experiment result.
\end{abstract}

DOI: 10.1103/PhysRevAccelBeams.21.060101

\section{INTRODUCTION}

New acceleration technologies for compact accelerators, such as laser-driven plasma wakefield accelerators, beamdriven plasma wakefield accelerators and structure-based wakefield accelerator (SBWAs), are rapidly developing many synergies. Development of laser plasma accelerators has made tremendous progress in generating soft $\mathrm{x}$-ray spontaneous undulator radiation and in pursuing development of compact free electron lasers (FELs). SBWAs or plasma wakefield accelerators with SRF injectors are good candidates for multiuser compact FELs with a high repetition rate [1].

The experimental study for these new acceleration technologies requires a large scale facility. But it is not realistic to construct a large scale facility for such a purpose. Therefore, the existing large facility must be considered for the experimental study on new acceleration technologies. Unfortunately, such an experimental study requires a long time without interruption to user operation (original purpose of facility). Therefore, conventional methods are not enough and the interleaving method between user operation and the experimental study on new acceleration technologies is encouraged.

A critical requirement to realize SBWAs is to extract maximum power up to $80 \%$ from drive bunches, and to obtain the highest energy for the witness bunch. A total of 4 times energy gain can typically be gained by witness electrons in SBWA, because the transformer ratio is 5 in the case of triangle distribution, assuming $80 \%$ efficiency. Some activities of SBWAs for compact $\mathrm{x}$-ray FELs are

\footnotetext{
tlssh@postech.ac.kr
}

Published by the American Physical Society under the terms of the Creative Commons Attribution 4.0 International license. Further distribution of this work must maintain attribution to the author(s) and the published article's title, journal citation, and DOI. being conducted in Argonne national laboratory [2] and the development and test of a $0.5 \mathrm{~m}$ long acceleration unit are planned in the linac extension area (LEA) tunnel downstream of the Argonne Advanced Photon Source (APS) injector linac [3].

The APS linac [4,5] is part of the injector complex of the APS storage ring. The thermionic rf electron gun (RG) provides the electron beam that is accelerated through the linac, injected into the particle accumulator ring (PAR), cooled, and transferred to the booster synchrotron. It is then accelerated, and injected into the APS storage ring. The linac is also equipped with a state-of-the-art S-band photocathode gun (PCG). Three fast-switching dipole magnets at the end of the linac (so-called interleaving dipoles) direct the electron beam in and out of the PAR and into the booster. Turning them off allows the beam to bypass the PAR and booster and direct electrons into the LEA tunnel that follows the APS linac. The LEA beam line is being configured for the testing of small-aperture apparatus and other beam physics experiments that will take advantage of the high brightness beam generated by the PCG.

Typically, the beam generated from RG is used $\sim 20$ seconds every two minutes to support storage ring top-up operation. A relatively quick switching between RG and PCG (interleaving) will allow to operate the LEA beam line during the rest of the two minutes. Because of the significantly different properties of beams produced by RG and PCG including beam energy, energy spread, bunch length, emittance and bunch charge, setting up one single lattice for the linac suitable for both beams is extremely challenging. In this paper we present a solution of this problem. Based on the initial study [6], we conceptually and experimentally demonstrated the solution of interleaving lattice in this paper. Section II introduces the optimum APS linac lattice for storage ring injection. Section III describes the optimum APS linac lattice for PCG and LEA 


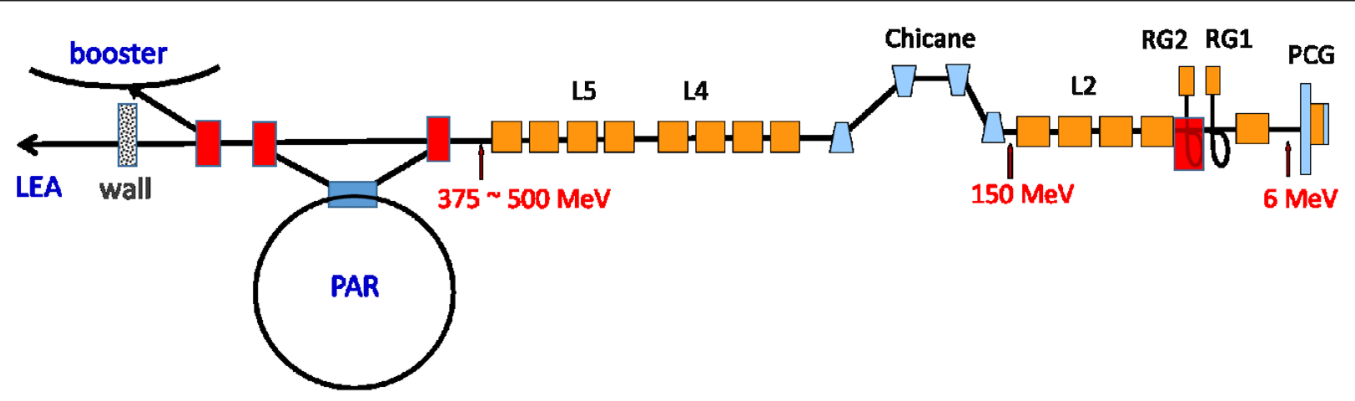

FIG. 1. Layout of APS linear accelerator.

experiments. A compromise interleaving lattice that conserves the high brightness of the PCG generated beam and maintains the high injection efficiency to PAR of the RG generated beam is described with experimental result in Sec. IV. Section V presents conclusions.

\section{APS LINAC FOR BEAM INJECTION}

The APS injector linac (Fig. 1) includes twelve S-band accelerating structures and an asymmetrical magnetic chicane that contains four dipoles and two quadrupoles [7]. A thermionic rf gun and an alpha magnet are used to inject a short train up to 17 electron microbunches into the linac. (A hot spare set of the thermionic rf gun and alpha magnet is also installed.) The PCG is installed at the front end of the linac and equipped with one additional accelerating structure upstream of the two thermionic rf guns. The chicane is not used for the compression of the RG generated beam as it is done by the alpha magnet, so the chicane is dedicated to compression of the PCG generated beam. Table I shows the main beam parameters.

The thermionic rf gun system includes the gun itself, the transport line from the gun to the entrance of the linac section, a fast kicker and alpha magnet. This system prepares an electron beam for injection into the APS storage ring. A tungsten dispenser cathode with a diameter of $6 \mathrm{~mm}$ produces an electron beam with up to $4.5 \mathrm{MeV}$ beam energy and up to $1.3 \mathrm{~A}$ peak macropulse currents. A fast kicker in the RG gun system limits the total current injected into the linac. An alpha magnet injects the beam from the rf gun into a chain of S-band accelerating structures. The alpha magnet also serves as a bunch compressor equipped with a low-energy particle scraper.

TABLE I. Parameter for APS linear accelerator.

\begin{tabular}{lcc}
\hline \hline Parameter & $\begin{array}{c}\text { RG beam for } \\
\text { injection }\end{array}$ & $\begin{array}{c}\text { PCG beam } \\
\text { for R\&D }\end{array}$ \\
\hline Beam energy $[\mathrm{MeV}]$ & 375 & 375 \\
Beam emittance $[\mu \mathrm{m} \mathrm{rad}]$ & 15 & 1 \\
Charge $[\mathrm{nC}]$ & 1.6 & 0.3 \\
Peak current $[\mathrm{kA}]$ & 0.3 & $>1$ \\
\hline \hline
\end{tabular}

The transport line consists of four quadrupoles before the alpha magnet and three quadrupoles after it.

An asymmetric chicane with the time-of-flight parameter $\mathrm{R}_{56}=-65 \mathrm{~mm}$ was originally designed to increase the peak current delivered to the low-energy undulator test line [5], and has been operated for the RG2 beam without bunch compression. To reduce emittance growth caused by the Coherent synchrotron radiation effect, an asymmetric configuration of the chicane and a small horizontal beta function at the exit of the last dipole magnet is used. A configuration with $R_{56}=-65 \mathrm{~mm}$ is less sensitive to difficult-to-control timing and phase errors [7]. Then the linac accelerating sections L2, L4 and L5 are adjusted to minimize the beam energy spread and obtain the desired final energy for beam injection into the PAR. The beam is typically accelerated on the crest of the rf phase in L2 because this beam is already compressed in the alpha magnet and satisfies the bunch length requirement for the beam injection into PAR. The dispersion function after the asymmetric chicane is closed by using two quadrupoles within the chicane system.

Since the requirement for injection into the PAR was satisfied, the APS linear accelerator has been operated with minimal beam loss through the linac after filtering lowenergy electrons in the alpha magnet. To prevent beam loss, beta functions though the linac are kept $\leq 25 \mathrm{~m}$. Two dispersion bumps (one in the chicane and one in the beam transport line from the linac to PAR) are present in the horizontal plane. Due to a large dispersion function and beta function in the region before the PAR, the trajectory is carefully controlled in this region to prevent beam loss. Lattice functions at the end point are matched to those at the PAR injection point.

\section{APS LINAC FOR ADVANCED ACCELERATOR R\&D}

A Linac coherent light source type photocathode gun [8] has been installed at the beginning of the APS linac to provide a low-emittance electron beam into the LEA beam line. The electrons are driven by a picosecond $\mathrm{Nd}$ :glass laser. The maximum field gradient in the gun cavity is $120 \mathrm{MV} / \mathrm{m}$. High brightness electron beam with peak current up to $1 \mathrm{kA}$ is prepared by first accelerating the electron beam to approximately $40-45 \mathrm{MeV}$ in the first 
accelerating section (L1), then by creating the energy chirp in the second accelerating section (L2) and finally by compressing the bunch in an asymmetric chicane. Accelerator sections (L4 and L5) located after the chicane are used to minimize the electron beam energy spread and to obtain the desired final beam energy.

The goal of a new beam line in the LEA tunnel of the APS is to deliver a high-brightness electron beam from the APS injector linac and the photocathode rf electron gun into the experimental area in the LEA tunnel for beam physics experiments. Two conditions are important for advanced accelerator R\&D: (1) the beam line optics must be tunable and capable of producing round and flat beams at the center of the experimental area (IP) where the device under the test (DUT) will be installed; (2) drift space in the experimental area must be sufficient to accommodate DUTs with up to $1 \mathrm{~m}$ length. The beta functions in the entire beam line should be kept less than $\sim 60 \mathrm{~m}$ and the total length of the beam line in the LEA tunnel must be less than $15 \mathrm{~m}$.

Another set of requirements is driven by a need to measure the effect of the DUT on the electron beam. Most of the DUTs are expected to produce an energy chirp along the electron bunch, and to kick the electrons along the bunch with variable strength in the horizontal plane. The energy chirp will be measured by a magnetic spectrometer installed at the end of the beam line. To measure the kick, we will map it to a coordinate at the location of the diagnostic YAG screen. Therefore, the part of the LEA lattice after the DUT is designed so that it converts the horizontal angle at the IP into the horizontal coordinate at the end of the beam line, and produces a relatively small vertical beam size at the same point. As examples, we consider two lattice solutions of the spectrometer and straight line for a round beam (Fig. 2). The betatron phase advance from the IP to the YAG screen at the end of the beam line is close to $\pi / 2$ in the horizontal plane, and is close to $\pi$ in the vertical plane. To measure the energy chirp with high-energy resolution $\left(2 \times 10^{-4}\right)$, a 40 -m vertical betatron function at the entrance of the bending magnet and



FIG. 2. Lattice functions of spectrometer beam line and straight beam line in LEA for round beam. 0.2-m vertical betatron function with 1.2-m dispersion function at the YAG screen are realized.

\section{INTERLEAVING LATTICE}

Because of the significantly different properties (e.g., energy, energy spread, bunch length, emittance, bunch charge) of beams produced by RG and PCG, setting up a single linac lattice that is suitable for both beams is an extremely challenging task. In this section, we will introduce interleaving lattice design with two different scheme. However, these schemes have the same longitudinal set condition and matching point. An emittance-measurement system is installed after the chicane (Fig. 1); this system consists of three beam-size measurement screens; the phase advance for each screen is matched to $60^{\circ}$. We use the location of this measurement system as the matching point for the interleaving lattice design, because Twiss functions can be also experimentally determined by using this measurement system. Longitudinal matching involves adjusting the phase and voltage of L2 for PCG beam to obtain the desired peak current and energy after the chicane, and to match with RG energy at the matching point. Then L4 and L5 are adjusted to minimize the energy spread and obtain the desired final energy for both beams. Energy gains for each beam (from RG and PCG) along the APS linac (Fig. 3) both have the same beam energy after accelerating section L2 and the same setting for L4 and L5 to gain the energy up to $375 \mathrm{MeV}$. Following longitudinal matching, transverse matching is done for both beams with two different schemes.

\section{A. Lattice design using the iterative matching procedure}

To see how the Twiss function for a beam can be diverged at a condition that has been optimized for a different beam, we investigate the change of the Twiss function after simply assuming that the beam line has been optimized for a different beam. During transformation along a beam transport line, the orientation and shape of

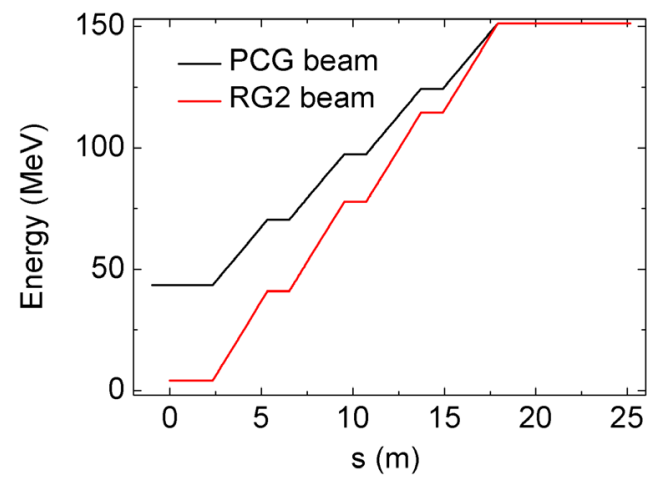

FIG. 3. Energy gain along APS linac. Black and red colors indicate PCG and RG2 beam, respectively. 


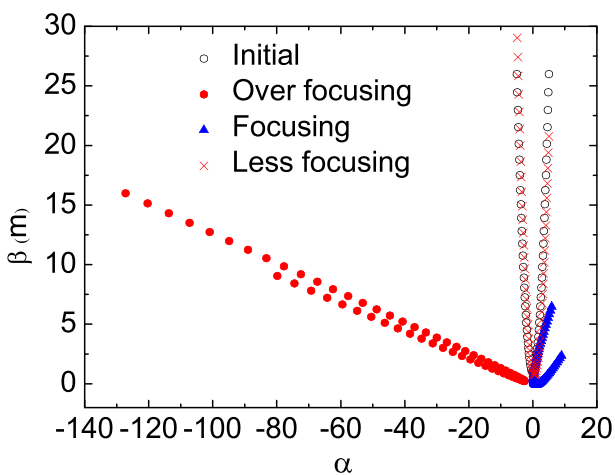

FIG. 4. Twiss functions for initial, over focusing, focusing and less focusing cases. Here over focusing is corresponding to lowenergy beam focusing with quadrupole set for high-energy beam.

the phase ellipse changes continuously but its area remains constant. In matrix formulation, the Twiss functions are [9]

$$
\left(\begin{array}{l}
\beta \\
\alpha \\
\gamma
\end{array}\right)=\left(\begin{array}{ccc}
m_{11}^{2} & -2 m_{12} m_{11} & m_{12}^{2} \\
-m_{11} m_{21} & m_{11} m_{22}+m_{12} m_{21} & -m_{12} m_{22} \\
m_{21}^{2} & -2 m_{22} m_{21} & m_{22}^{2}
\end{array}\right)\left(\begin{array}{c}
\beta_{0} \\
\alpha_{0} \\
\gamma_{0}
\end{array}\right),
$$

where $m$ is a transfer matrix element for the particle tracking:

$$
\left(\begin{array}{c}
x \\
x^{\prime}
\end{array}\right)=\left(\begin{array}{ll}
m_{11} & m_{12} \\
m_{21} & m_{22}
\end{array}\right)\left(\begin{array}{c}
x_{0} \\
x_{0}^{\prime}
\end{array}\right)
$$

If we consider a quadrupole doublet with focusing relation $f_{1}=-f_{2}=f$ and center-to-center distance $d$ between two quadrupoles, transfer matrix elements are given by

$M=\left(\begin{array}{ll}m_{11} & m_{12} \\ m_{21} & m_{22}\end{array}\right)=\left(\begin{array}{cc}1-\frac{d}{f} & d \\ -\frac{d}{f^{2}} & 1+\frac{d}{f}\end{array}\right), \quad \frac{1}{f}=\sqrt{k} \sin \sqrt{k} L$,

where $L$ is quadrupole length, $k=\frac{g}{B \rho}$ when focusing is horizontal, and

$$
g=\frac{d B_{x}}{d y}=\frac{d B_{y}}{d x}=\frac{B_{0}}{a}
$$

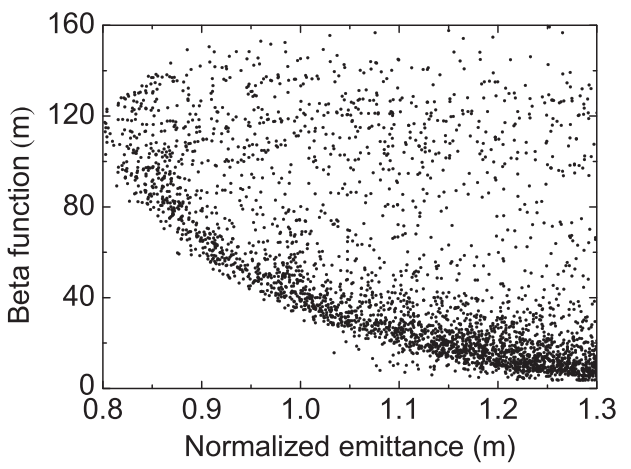

FIG. 6. Results from MOGA: Beta functions vs normalized emittance.

is the field gradient where $B_{0}$ is magnetic field at the pole tip $(r=a)$ [9]. The Twiss function of a low-energy beam degrades dramatically through a quadrupole doublet that is focused appropriately for a high-energy beam (Fig. 4). Therefore, in interleaving lattice design the quadrupole through the linac must be set specifically for a low-energy beam to keep a small Twiss function.

Small Twiss functions for the PCG beam should be satisfied at the exit of the photoinjector system (Fig. 5, starting point) to keep small Twiss functions for a highenergy beam through the linac, because the high-energy beam passes through a low quadrupole focusing system like drift space (Fig. 4). Therefore, some parameters (e.g., laser spot size, solenoid field, gun rf phase, field gradient for the first rf accelerator) in the photoinjector system are fully explored to achieve small emittance and Twiss functions (at the starting point in Fig. 5) by using genetic optimization with ASTRA [10] simulation. Results of a genetic optimization [11] are shown in Fig. 6. Here a tradeoff between the achievable emittance and the maximum values of the beta functions is shown (Twiss functions and emittance in $\mathrm{x}$ and $\mathrm{y}$ planes that are almost the same, because all components are cylindrically symmetric in this simulation). For example, accepting the normalized emittance of $1.2 \mu \mathrm{m}$ will allow to reduce beta functions up to $5 \mathrm{~m}$ by reducing the laser spot size on the cathode. However, when the emitting spot size on the cathode becomes $<0.25 \mathrm{~mm}$, the space charge effects become too strong preventing beam emission of the desired bunch charge. The longitudinal space charge effect increases as beam size decreases, and initial particles in the tail part

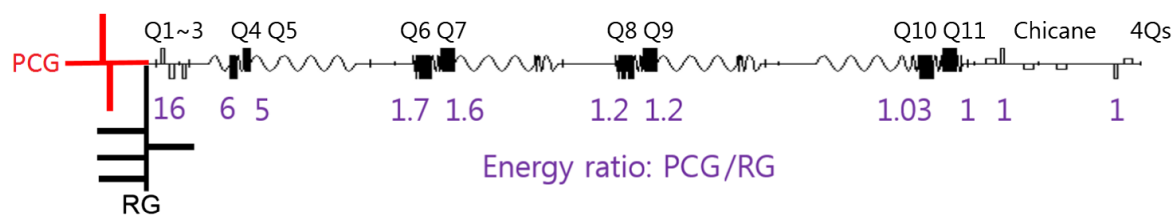

FIG. 5. Beam line layout from each gun to the matching point downstream of the chicane. Energy ration between PCG and RG is also shown in the figure. 




(c)

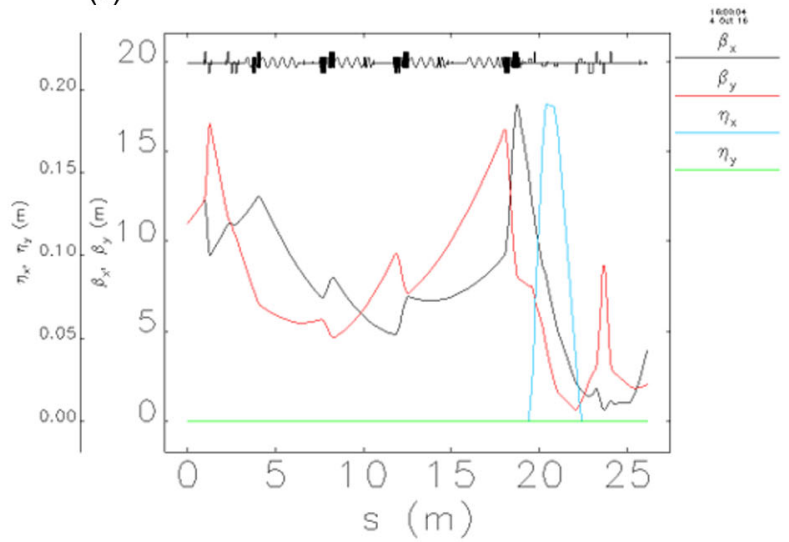

(e)

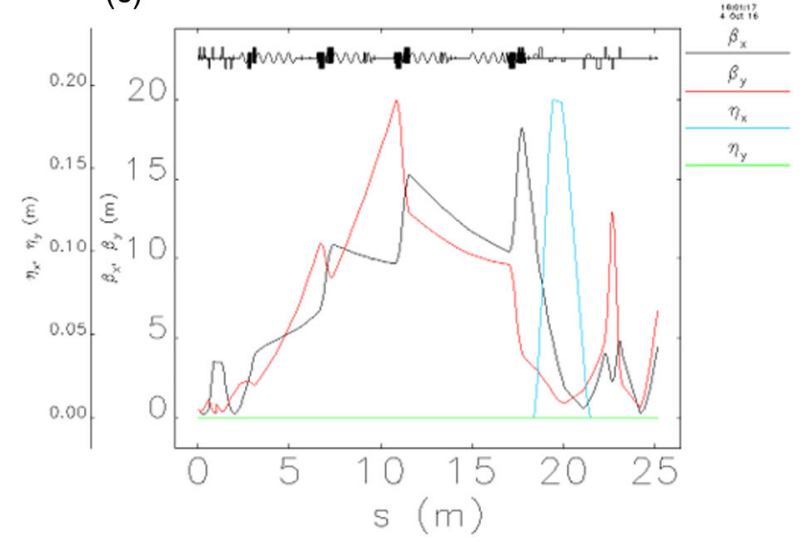

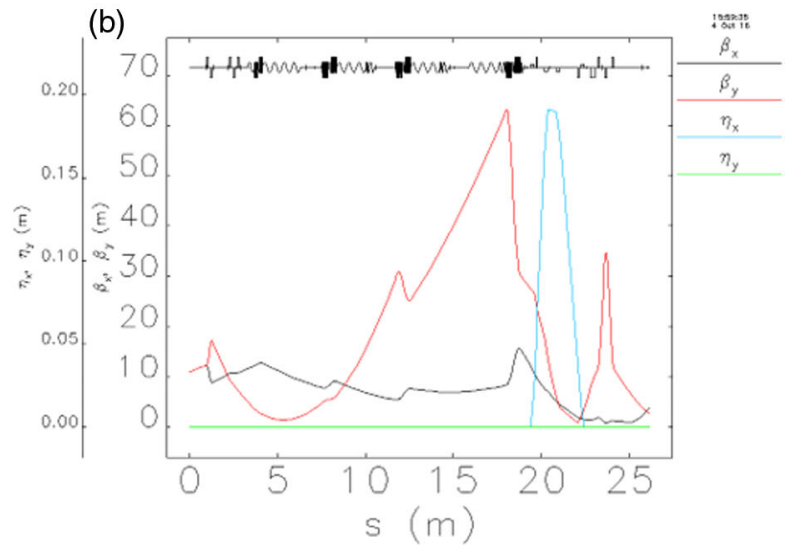

(d)



(f)



FIG. 7. The results along the process of interleaving lattice design. (a) RG beam optimization. (b) PCG beam result by setting quadrupoles obtained in (a) to PCG. (c) PCG optimization using two initial dedicated quadrupoles for PCG. (d) PCG optimization using Q11 and 4Qs for emittance measurement. (e) RG beam result by setting Q11 and 4Qs obtained in (d) to RG. (f) RG optimization using four initial dedicated quadrupoles for RG.

cannot overcome repulsive space charge force despite the high rf field in the gun cavity.

Figure 5 shows the layout up to the matching point and energy ratio between the PCG beam and the RG2 beam. The following sequence of steps is executed to achieve transverse matching: (i) optimizing the lattice for the beam generated by the RG (this step sets the quadrupole for the low-energy beam based on the result of Fig. 4); (ii) setting these quadrupole values for the PCG beam with energy scaling; (iii) optimizing the lattice for the beam generated by the PCG [this step uses two dedicated quadrupoles shown (Fig. 5, red bars) with the goal of keeping the beta functions in the linac low]; (iv) optimizing the PCG beam using Q11 to minimize the horizontal beta function at the end of the chicane to reduce the CSR effect [7]; (v) matching the diagnostics condition for emittance measurement 
using the four quadrupoles (Fig. 5, "4Qs"); and (vi) retuning the RG beam with four dedicated quadrupoles shown (Fig. 5, black bars) to match to the same lattice functions after the chicane as in the case of the PCG lattice.

Finally, the lattice functions for both RG and PCG beams become the same after the matching point, because the beam energy and lattice functions at the matching point after the chicane are the same. The lattice functions change at each step during the process of interleaving lattice design (Fig. 7). Figures 7(d) and 7(f) show the final beta functions in the interleaving lattice for PCG and RG beams up to the matching point, respectively. All constraint conditions for the matching are fully satisfied and lattice functions are each close to their dedicated optimal values.

\section{B. Simultaneous matching for both beams}

ELEGANT was used to achieve simultaneous matching of both beams for the interleaving lattice design. The beam line for interleaving lattice design is defined as (beam line for RG beam, mark 1, energy varying, Twiss varying, beam line for PCG beam, mark 2) in ELEGANT input. Here mark 1 and mark 2 are the matching points for the RG beam and the PCG beam, respectively. The initial Twiss function and beam energy were considered for the RG beam, then the Twiss function and beam energy were varied to the initial values for the PCG beam. As a matching condition, beta functions and alpha functions are the same at mark 1 and mark 2 positions, and beta functions through all beam lines were kept $<100 \mathrm{~m}$. Instead of geometric strength, bore radius and pole tip field for quadrupole parameters [Eq. (4)] are used as the matching variable for the energy independent quadrupole set value. A hybrid-simplex optimization algorithm was used in a parallel computing system. Beta functions for both beams (Fig. 8) were obtained by simultaneous matching. Beta functions were kept $<100 \mathrm{~m}$ through each beam line, and the same beta functions were matched at the end point. Fortunately, beta functions for the RG beam were kept relatively small up to $5 \mathrm{~m}$ from the gun, where large beam size is assumed.

\section{Preliminary experiment study}

This section describes the initial experimental result in realizing interleaving lattice design. For the experiment study, we considered the iterative scheme used in interleaving lattice design, because the minimum change of operation condition for the RG beam is required and all requirements on the PCG beam are fulfilled. To demonstrate interleaving lattice design, the preliminary experiment study was performed with limited control knobs for Twiss function and emittance from PCG. Laser spot size for PCG and dedicated quadrupoles on the first accelerator section will be available later. The main purpose in the experiment study is to realize $100 \%$ beam transmission for the PCG beam, and the same Twiss function with the RG2 beam at the matching point. Dedicated quadrupoles (Fig. 5, (a)
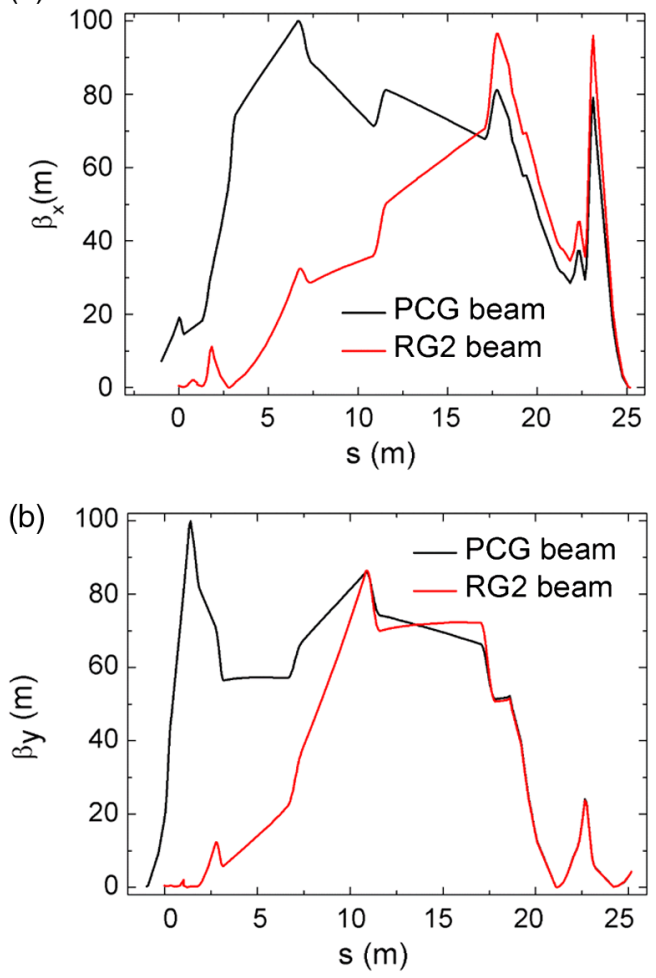

FIG. 8. (a) Horizontal and (b) vertical beta functions for RG beam (black color) and PCG beam (red color). Beta functions for both beams are the same at the end point. Note that starting positions for both beams are not consistent due to different beam line lengths.

red bar) and corrector for the PCG beam are adjusted to achieve the main goal.

Following acceleration in the linac and chicane, a threescreen emittance measurement system is used to both the emittance and Twiss parameters (Table II). Emittance is determined by an rms fit to the beam distribution measured at the three screens. An optical filter is used to prevent signal saturation on the screens. With only dedicated quadrupoles used for the PCG beam, Twiss functions of the beam are close to those of the RG2 beam in the matching section. The same operation conditions are used for both beams after the emittance measurement system because both beams have similar energy and Twiss functions at emittance that point. Matching quadrupoles can be used to optimize emittance and Twiss functions of the PCG beam [Fig. 7(d)]. Measured emittance of the PCG beam

TABLE II. Result for emittance and Twiss function measurement.

\begin{tabular}{lccc}
\hline \hline Parameter & $\beta_{x} / \beta_{y}$ & $\alpha_{x} / \alpha_{y}$ & $\varepsilon_{x} / \varepsilon_{y}$ \\
\hline RG beam & $2.06 / 3.27$ & $1.56 / 1.38$ & $17.49 / 13.74$ \\
PCG beam & $3.83 / 3.62$ & $3.2 / 3.15$ & $3.85 / 3.9$ \\
PCG beam (matched) & $2.72 / 2.94$ & $1.95 / 2.01$ & $2.71 / 3.98$ \\
\hline \hline
\end{tabular}


(a)

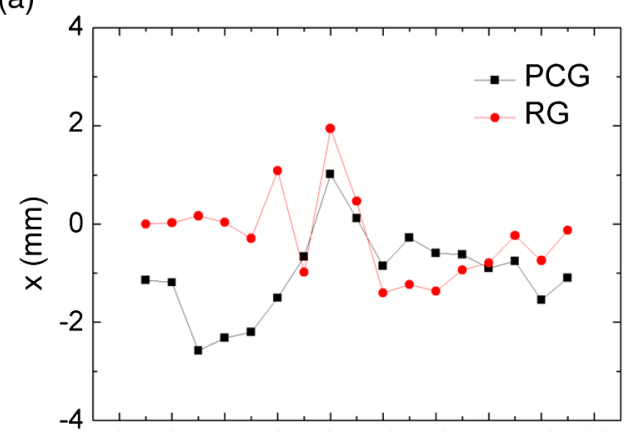

(b)

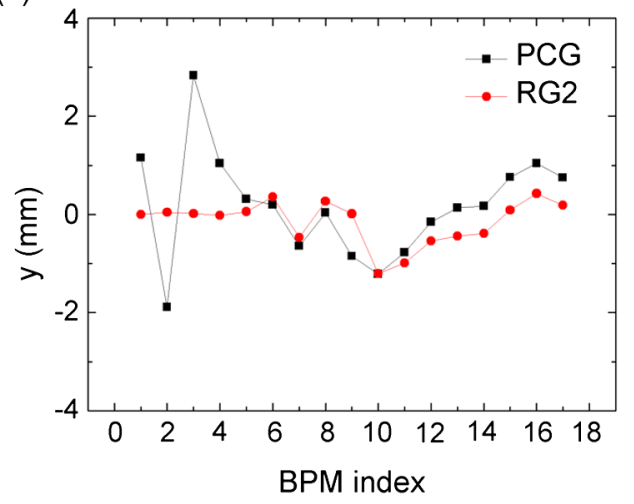

FIG. 9. (a) Horizontal and (b) vertical beam trajectories for PCG and RG2 beam at the same corrector set configuration.

was larger than design value (Table II), so it will be reduced if a gun laser with small spot size is used.

We have considered lattice functions for two beams, but the trajectory of each PCG beam can be significantly distorted in the corrector set values for the RG beam, because the two beams have different energies. Fortunately, the trajectory of the PCG beam can be acceptable without iteratively correcting both RG2 and PCG beams (Fig. 9); transmission of RG2 and PCG beams are up to $90 \%$ and $100 \%$, respectively. Normal transmission of the RG2 beam during operation is up to $90 \%$.

\section{CONCLUSION}

We have described the lattice design and preliminary experiment results for the APS injector linac in support of interleaving of the thermionic rf electron gun and photocathode rf gun. Despite significant differences of the original beams, an excellent compromise was found in simulation and realized in a preliminary experimental study. This compromise allows efficient injection of the high-charge RG2 beam into the particle accumulator ring, and efficient transport of the high-brightness PCG beam to the experimental beam line in the linac extension area for testing of small aperture apparatus and other beam-physics experiments. As future work, we will continue the interleaving lattice optimization with additional quadrupoles installed for PCG beam front-end beta-function control. An advanced accelerator R\&D experiment will be conducted in LEA with the linac operating in interleaving mode. This work is the first study for interleaving operation and will be good reference for existing accelerator facilities to realize and test advanced accelerator concepts and hardware.

\section{ACKNOWLEDGMENTS}

We wish to thank L. Emery, V. Sajaev, and N. Sereno for providing helpful information and the many useful discussions. This research was supported by the U.S. Department of Energy, Office of Science, under Contract No. DE-AC0206CH11357. This research was also supported by the Basic Science Research Program through the National Research Foundation of Korea (NRF-2015R1D1A1A01060049).

[1] C. Jing, J. G. Power, and A. Zholents, Dielectric wakefield accelerator to drive the future FEL light source, in Proceedings of the 2nd International Particle Accelerator Conference, San Sebastián, Spain (EPS-AG, Spain, 2011), pp. 1485-1487.

[2] C. Jing, A. Kanareykin, J. G. Power, M. Conde, W. Liu, S. Antipov, P. Schoessow, and W. Gai, Experimental Demonstration of Wakefield Acceleration in a Tunable Dielectric Loaded Accelerating Structure, Phys. Rev. Lett. 106, 164802 (2011).

[3] A. Zholents (unpublished).

[4] R. Soliday, S. Pasky, and M. Borland, Automated operation of the APS linac using the procedure execution manager, in Proceedings of the 20th International Linac Conference, LINAC-2000, Monterey, CA, 2000 (SLAC, Menlo Park, CA, 2000), pp. 524-526.

[5] S. Milton et al., Exponential gain and saturation of a self-amplified spontaneous emission free-electron laser, Science 292, 2037 (2001).

[6] S. Shin, Yin-E. Sun, and A. Zholents, Interleaving lattice design for APS linac, in Proceedings of NAPAC2016, Chicago, 2016, pp. 713-715.

[7] M. Borland, Design and performance simulations of the bunch compressor for the Advanced Photon Source LowEnergy Undulator Test Line free-electron laser, Phys. Rev. ST Accel. Beams 4, 074201 (2001).

[8] Y. Sun et al., Commissioning of the photo-cathode RF gun at APS, in Proceedings of FEL 2014, Basel, Swizerland, 2014, pp. 803-806.

[9] H. Wiedemann, Particle Accelerator Physics (Springer, New York, 2007).

[10] K. Flottmann, ASTRA user manual, URL: www.desy.de/ $\sim$ mpyflo/Astra documentation.

[11] M. Borland and H. Shang (unpublished). 